\title{
ON THE EXISTENCE OF CURVES WITH ASSIGNED SINGULARITIES *
}

\author{
BY J. L. COOLIDGE
}

The fundamental relations which connect the point and line singularities of algebraic plane curves are called Plücker's equations; they give necessary limitations upon the numbers of characteristics of the different sorts. These equations do not, however, contain in themselves any existence theorems, and after a solution in integers has been obtained, there is no guarantee that there is any curve whose characteristics are the numbers found. Erroneous views have been held on this point. The fact that a quartic is possible with three cusps suggests the existence of a unicursal curve of any order all of whose singular points are cusps; such a curve can not exist.

Various attempts have been made to show the existence of curves with assigned singularities, the most important being that of Lefschetz. $\dagger$ As far as simple nodes are concerned, he completed the solution, exhibiting the existence of plane curves with no singularities but simple nodes, and these in any desired number up to the theoretical maximum. He also showed that the requirement of each additional node imposed just one new condition. With regard to cusps, he was less successful. He established certain upper limits which may be attained, but the conclusions are not clean-cut, and depend upon what he calls the Postulate of singularities, which consists essentially in assuming that when we require a certain curve to have an additional cusp, we do not thereby impose upon it automatically more than one additional cusp.

The present paper follows closely Lefschetz's methods, but reaches a more definite and much simpler conclusion, and does so without the use of his postulate, which is proved in the course of the work. The final result is as follows.

* Presented to the Society, December 27, 1922.

$\dagger$ On the existence of loci with given singularities, TRANSACTIONS OF THIS SocIETY, vol. 14 (1913), p. 23. 
Theorem. The maximum possible number of cusps for a curve of odd order $n$ and deficiency $p$, where $n \geqq 2 p+\sqrt{8 p+9}$, is

$$
\frac{3}{2}[(n-2)+2 p]-\frac{1}{2} \text {. }
$$

The maximum number for a curve of even order $n$ and deficiency $p$, where $n \geqq 2 p+1+\sqrt{8 p+1}$, is

$$
\frac{3}{2}[(n-2)+2 p] \text {. }
$$

There exist curves of order $n$ and deficiency $p$ having cusps in any number up to the maximum.

Let us begin by writing some of Plücker's numbers, and the equations which connect them:

Order of curve $=n, \quad$ Number of cusps $=\kappa$,

Class of curve $=m, \quad$ Deficiency $=p$,

Number of nodes $=\delta, \quad$ Number of inflections $=i$.

We have, then, the following equations of Plücker:

$$
\begin{aligned}
m & =n(n-1)-2(\delta+\kappa)-\kappa, \\
p & =\frac{(n-1)(n-2)}{2}-(\delta+\kappa), \\
m & =2(n-1)+2 p-\kappa, \\
n & =2(m-1)+2 p-i, \\
i & =3 n(n-2)-6(\delta+\kappa)-2 \kappa, \\
i & =3[(n-2)+2 p]-2 \kappa, \\
\kappa & =\frac{3}{2}[(n-2)+2 p]-\frac{i}{2} .
\end{aligned}
$$

In order to prove that a curve may have the maximum number of cusps we must show that we can have a curve of given order and deficiency with one inflection, if the order be odd, and no inflection if it be even.

Suppose that we have a unicursal curve of order $n$ with a multiple point $A$ of order $n-1$, all the tangents being distinct. Let $B$ be a simple point of this curve. Pass a quadric surface through $A$ and $B$, and let $V$ be the point where a generator through $A$ meets a generator through $B$. Let us project our 
plane curve upon this quadric from $V$. The space curve will not pass through $V$, but will meet $V A$ at the points of contact of the tangent planes through the tangents to the plane curve at $A$, and will meet $V B$ in the point of contact of the tangent plane through the tangent at $B$ to the plane curve. Since the space curve does not go through $V$, and meets a plane through $V$ in $n$ points other than $V$, it is a space curve of order $n$. Now if we project this space curve back upon the plane from a point which does not lie on one of its tangents, or on the common secant of two intersecting tangents, we get a plane curve, also of order $n$, whose only singularities are nodes, and this plane curve is unicursal, for it is birationally related to the first one. But if the point of projection lie upon a tangent to the space curve the new plane curve will have just one cusp, but a deficiency 0 as before. We see, therefore, that we may have a unicursal curve with no singularities but nodes, ${ }^{*}$ or with a single cusp. The classes in these two cases are, by (3),

$$
2(n-1) ; \quad 2(n-1)-1 .
$$

If we increase $n$ to $n+1$, we shall get the classes

$$
2 n ; \quad 2 n-1 .
$$

Thus it appears that we can find a unicursal curve with one cusp and any odd class above 1, or with no cusp and any even class. Dually, we can find a curve which is unicursal with one inflection and any odd order, or with no inflection, and any even order, and this shows that we can find a unicursal curve with the maximum number of cusps. Now for a curve of deficiency $p$, we follow a general form of reasoning of Lefschetz.

There will exist a curve of deficiency $p$ and the maximum number of cusps if there exist a curve of given order and deficiency with one inflection or no inflection, as the case may be, i.e., if there exist a curve of given deficiency and class with one cusp or no cusp, or, finally, a curve of given order

* See Snyder, Construction of curves of given deficiency, this Bullerin, vol. 15 (1908), p. 1. 
and deficiency with one cusp or no cusp. Since the number of double tangents cannot be negative, when $m$ and $n$ are even, $i=0$, and

$$
\begin{gathered}
(m-1)(m-2)>2 p, \quad 2(m-1) \geqq 1+\sqrt{8 p+1}, \\
n \geqq 2 p+1+\sqrt{8 p+1} ;
\end{gathered}
$$

when $n$ is odd, $i=1$, and

$$
n \geqq 2 p+\sqrt{8 p+9} \text {. }
$$

What is the contrary hypothesis? It is that if a curve have deficiency $p$ and given order it must have more than one cusp or the equivalent in higher singularities. If this were true for every curve of deficiency $p$ it would be true for every curve of lesser deficiency, for the curves of lesser deficiency have more singularities, i.e., they have imposed upon them all the conditions for the curves of higher deficiency, and others also. But this last hypothesis does not hold where the deficiency is 0 ; hence it does not hold in the general case. Thus, there are curves of any deficiency with one cusp or no cusps, or with one inflection or no inflections, hence curves of any deficiency with the maximum number of cusps.

We have now, lastly, to prove that we can have any number of cusps less than the maximum. We can have no cusps at all or one cusp. Start with this and impose conditions for one cusp, then another, etc. We shall thus get any number of cusps up to the maximum unless it happen that at some stage of the process the imposition of an additional cuspidal condition will produce several new cusps. If this should happen, the intervening number of cusps would be impossible. But another result would be reached also, for the number of additional conditions imposed in order to change from a curve with no cusps or one cusp to one with the maximum number of cusps would be less than the number of cusps so gained.

If a curve have the order $n$ and $\kappa$ cusps, where this number is one or zero, according as $n$ is odd or even, the number of degrees of freedom is

$$
\frac{n(n+3)}{2}-\left[\frac{(n-1)(n-2)}{2}-p\right]-\kappa=3 n+p-1-\kappa .
$$


Hence the number of degrees of freedom of a curve of class $m$ with one inflection or no inflections is

$$
3 m+p-1-i,
$$

where $i=0$ or $i=1$.

If the conditions for cusps are all independent, and only in that case, the amount of freedom would be

$$
\begin{aligned}
\frac{n(n+3)}{2}-\left[\frac{(n-1)(n-2)}{2}-p\right] & \\
& -\frac{3}{2}[(n-2)+2 p]+\frac{i}{2}=\frac{3 n}{2}+2-2 p+\frac{i}{2} .
\end{aligned}
$$

But

$$
\begin{aligned}
m=2(n-1)+2 p-\frac{3}{2}[(n-2)+2 p]+ & \frac{i}{2} \\
& =\frac{n}{2}+1-p+\frac{i}{2} .
\end{aligned}
$$

Hence

$$
\frac{3 n}{2}+2-2 p+\frac{i}{2}=3 m+p-1-i .
$$

It appears, then, that a curve with the maximum number of cusps has exactly the freedom compatible with the appearance of one new cusp for each additional cuspidal condition, and with no other hypothesis. This proves Lefschetz' postulate of singularities, and shows that we may assign any number of cusps up to the maximum, without fear that any case is impossible, i.e., proves our theorem in its entirety.

HARVARD UNIVERSITY 